\title{
¿Egocentrismo o altruismo en la adolescencia? Un estudio empírico sobre los estudiantes de colegios Waldorf, cristianos y públicos ${ }^{1}$
}

\section{Egocentrismo ou altruísmo na adolescência? Um estudo empírico sobre os estudantes de escolas Waldorf, confessionais cristãs e públicas}

\section{Egocentrism or Altruism in Adolescents? An Empirical Study on Students in Waldorf, Christian and State Schools}

\author{
Axel Föller-Mancini² \\ Peter Heusser ${ }^{3}$ \\ Arndt Büssing ${ }^{3}$
}

\begin{abstract}
RESUMEN
Este artículo presenta una síntesis de un estudio sobre los posicionamientos egocéntricos en la adolescencia realizado en tres contextos escolares diferentes. El análisis permitió determinar que el posicionamiento egocéntrico puede ser mejor determinado a través de dos predictores: el sentido del propio bienestar (eudemonismo) y la convicción de que "la lástima ajena previene
\end{abstract}

DOI: $10.1590 / 0104-4060.41489$

1 Este artículo se basa en el estudio empírico publicado en la Revista RoSE: Research on Steiner Education: Self-centredness in Adoldescents: An empirical study of students of Steiner schools, Christian academic high schools, and public schools. v. 1, n. 2, p. 148-164, 2010. Esta versión corresponde a una actualización teórica. Traducción de Aida Montenegro.

2 Universidad Alanus de Artes y Ciencias Sociales. Departamento de Educación. Alfter, Alemania. Villestraße 3, 53347.

3 Universidad de Witten / Herdecke. Facultad de Salud. Witten, Alemania. Alfred-Herrhausen-Straße 50, 58448. 
tomar iniciativas propias". Estos dos factores fueron correlacionados con la satisfacción del contexto escolar específico, la orientación espiritual y la idea de ayudar a otros. Los posicionamientos y los ideales éticos difieren entre los estudiantes de los tres contextos escolares: colegios Waldorf, colegios cristianos y colegios estatales. Los resultados confirman que la tendencia egocéntrica fue significativamente inferior en el grupo femenino de adolescentes. Este artículo invita a reflexionar sobre el papel que juega el contexto escolar en el proceso de desarrollo de la etapa adolescente a la adulta de los estudiantes.

Palabras clave: egocentrismo; adolescencia; altruismo; Rudolf Steiner.

\title{
RESUMO
}

Este artigo apresenta uma síntese de um estudo sobre os posicionamentos egocêntricos na adolescência realizado em três contextos escolares diferentes. A análise permitiu determinar que o posicionamento egocêntrico está relacionado a dois fatores: o sentido do próprio bem-estar (eudemonismo) e a convicção de que "a compaixão evita a busca por iniciativas próprias". Estes fatores foram correlacionados com a satisfação no contexto escolar específico, a orientação espiritual e a ideia de ajudar os outros. Os posicionamentos e os ideais éticos diferem entre os estudantes dos três contextos escolares: escolas Waldorf, escolas cristãs e escolas públicas. Os resultados confirmam que a tendência egocêntrica foi significativamente inferior no grupo feminino de adolescentes. Este artigo convida à reflexão acerca do papel do contexto escolar no processo de desenvolvimento da etapa adolescente à adulta dos estudantes.

Palavras-chave: egocentrismo; adolescência; altruísmo; Rudolf Steiner.

\begin{abstract}
This article presents a summary of a study on egocentric positionings in adolescents conducted in three different school contexts. The analysis reveals that egocentric positioning can be better determined through two predictors: a sense of personal well-being (eudemonism) and a conviction that "shame experienced on behalf of another person prevents oneself to take personal initiatives". These two factors correlate to satisfaction within the school context, spiritual orientation and the idea of helping others. The positionings and the ethical ideas differ from the students of the three school contexts: Waldorf, Christian, and State schools. The results confirm that the egocentric tendency was significantly inferior in the female group of adolescents. This article invites readers to reflect upon the role that the school context plays in the process of development of the students from adolescence to adultness.
\end{abstract}

Keywords: egocentrism; adolescence; altruism; Rudolf Steiner. 


\section{Posicionamiento egocéntrico en la adolescencia}

El sociólogo Peter Berger anunció en 1975 una "insatisfacción en la modernidad", a causa de una "pérdida metafísica de origen". (BERGER, 1975). Otros autores, en el siglo XX, diagnosticaron un incremento de la emancipación sobre la tradición y las costumbres, lo cual posteriormente encaminaría al estudio biográfico y al desarrollo social. Así, aspectos como la religión, el código moral y el modelo familiar son determinantes en el individuo. Cada persona será libre y se transformará a través de la confrontación como ser individual. Así, por ejemplo, la comprensión de una vida exitosa y feliz no será buscada en la literatura o entendida a través de la orientación de los padres. Cada estadio del desarrollo biográfico será comprendido desde la tendencia a la individualización. Erikson (1973), en su estudio de psicología del desarrollo sobre el ideal de un americano masculino promedio, describió la imagen del desarrollo de los adolescentes como moratorio o umbral. En esta situación moratoria se presenta la formación de la crisis interna hacia la identidad estable.

En la actualidad otro modelo se discute: La adolescencia como una situación de umbral universal, en el sentido de moratorio prolongado. En el proceso de comunicación global se entiende al individuo con inspiraciones momentáneas, que se autodefinen nuevamente. La perspectiva interna y externa están sujetas a un cambio permanente. Una parte de la identidad temporal se desvanece y nuevos aspectos de la personalidad aparecen. (BOBERT, 2007). Conforme a esto, la adolescencia postmoderna necesita una mejor estructura estable de desarrollo y competencias especiales con las cuales pueda enfrentar cada período de la vida.

Las competencias de desarrollo juegan un papel importante. Cuatro competencias claves son sugeridas por el investigador educativo Hurrelmann (2010). El perfil típico ideal de estas competencias son: intelectualidad diferenciada con habilidades sociales; formación del papel del género independiente y las relaciones sociales cercanas; la habilidad de administrar el dinero y otros asuntos financieros; $y$, el desarrollo de la asignación de recursos y la participación en debates políticos. (HURRELMANN, 2010). Este perfil puede ser caracterizado por un estado de inconsistencia debido a la influencia de las condiciones de cambio social. Es así como los adolescentes establecen nuevas formas de relacionarse y de posicionarse de manera egocéntrica. Desde esta perspectiva, se puede deducir que hay una variación del concepto de adolescencia como estado de transición.

La dinámica de la individualización en la adolescencia promovida por la sociedad resalta la independencia temprana del adolescente, y a su vez, la toma de responsabilidad propia. Cuando estas cualidades se combinan pueden ofrecer una 
“oportunidad para planear, si es necesario, implementar el desarrollo personal y la biografía libre de las ataduras sociales y de la independencia de las raíces sociales, así en comunión con sus intereses individuales y las demandas, necesidades y metas propias". (MANSEL, 1995, p. 18). La oportunidad de orientar en valores a los estudiantes debería ser un efecto colateral de la intención de la autoridad socializadora que es la escuela. (FEND, 1976; ROLFF, 1997). Cada ambiente escolar con su cultura particular presenta la posibilidad de un análisis comparativo de las actitudes de los adolescentes. Desde esta perspectiva, este estudio exploró este concepto en tres contextos escolares: colegios académicos cristianos, colegios Waldorf (Steiner), y colegios públicos convencionales.

Los colegios cristianos y Waldorf se identifican como colegios de auto-desarrollo con la visión cultural no materialista de sus estudiantes, y la participación activa de los padres de familia. Estos dos contextos escolares son parte del llamado tercer sector educativo. Por otro lado, las escuelas públicas no se ubican en un contexto filosófico, cultural o religioso específico, proclamando así un enfoque educativo neutral.

En una investigación previa sobre espiritualidad con adolescentes, se determinaron aspectos no formales en términos de conciencia relacional de relevancia considerable. (BÜSSING, 2010b). También se asoció negativamente la interacción consciente y la convicción trascendente con el posicionamiento egocéntrico, con un valor de $52 \%$ en el género masculino. Una variedad de preguntas emergen con respecto a qué factores pueden influir en el posicionamiento egocéntrico entre los adolescentes. Por ejemplo, factores culturales, escolares, familiares y/o religiosos.

La importancia de las necesidades religiosas o espirituales y su impacto en la forma de afrontar la vida, al igual que la prevención de enfermedades, son temas relevantes actualmente en el campo de investigación. (SEYRINGER, 2007; BÜSSING, 2009, 2010a, 2010b). Uno de los objetivos de este estudio fue identificar y comparar el posicionamiento egocéntrico y la tendencia altruista de tres grupos de adolescentes en tres colegios.

\section{Colegios con enfoque cristiano}

Los criterios de calidad de los colegios cristianos se formulan teniendo en cuenta las directrices de la conferencia alemana de obispos. Las directrices se centran en: (a) la convicción básica de la dignidad personal del niño y el adolescente basada en la firme creencia de que Dios creó el hombre a su imagen 
y semejanza; (b) el esfuerzo para alcanzar la interiorización de la creencia, la cultura y la vida; (c) el principio general de la comunidad educativa, un principio que puede ser concebido como la consecuencia del entendimiento de la educación y la aspiración que permite que la organización de la vida escolar guíe las convicciones básicas de la creencia de la iglesia. La enseñanza en estos colegios tiene la misión clara de permitir el desarrollo de la personalidad y la formación de los valores que guíen los ideales cristianos.

\section{Colegios Waldorf (Steiner)}

Los colegios Waldorf o Steiner presentan un programa educativo autónomo basado en una didáctica diferenciada y específica a la asignatura. Los colegios Waldorf están representados a nivel internacional. Actualmente, hay 1.056 colegios Waldorf en 60 países. Ello demuestra que esta educación puede entenderse en cada contexto social y cultural. (BUND DER FREIEN WALDORFSCHULEN, 2014). El método se centra en el niño como ser individual y espiritual que puede ser inspirado en su propio desarrollo. La pedagogía y la educación Waldorf apoyan el potencial de cada estado de desarrollo donde la independencia y la emancipación se convierten en experiencias valiosas para el alumno. La dimensión espiritual no se evidencia en el contenido, sino en la forma en que ellos son enseñados. La enseñanza Waldorf emplea métodos enfocados en los aspectos físicos, artísticos y emotivos de los niños y motiva la intuición para alcanzar tanto como sea posible las bases de su propio desarrollo. (SCHIEREN, 2010).

Con respecto a la posición y orientación de vida de los estudiantes Waldorf, investigaciones realizadas por Randoll (1997) y un estudio de retrospectiva sobre ex-alumnos Waldorf (BARZ; RANDOLL, 2007) revelan la ausencia de auto-suscripción específica. De manera contraria, sobresalen características como el espíritu abierto, la tolerancia y el compromiso social. La ambición y la imposición se presentaron en un grado más inferior. (BARZ; RANDOLL, 2007). Un estudio realizado por el Instituto de Criminología en la Baja Sajonia revela que los estudiantes Waldorf presentaron el menor nivel de xenofobia y rechazo por las personas de pensamiento diferente, en comparación con otros contextos escolares. (BAIER, 2008).

Las investigaciones anteriores permiten asegurar que la posición de los estudiantes y su procedencia social son aspectos a tener en cuenta. Sin embargo, los autores de estos resultados no dejan conclusiones sobre el efecto resultante 
de la pedagogía implementada. Estos resultados pueden estar relacionados con la cuenta del capital cultural. Bourdieu (1983) entiende este concepto de los beneficios y recursos culturales de cada generación como la transmisión del sistema de regulación, la orientación normativa y la cualificación, lo cual contribuye a la estabilidad de la cultura. Los recursos culturales cuentan como modelos de pensamiento y valoración que en el transcurso de la socialización serán interiorizados. En el caso de la preferencia profesional, Bonhoeffer, Brater y Hemmer-Schanze (2007) señalan, en su estudio sobre ex-alumnos Waldorf, que la preferencia corresponde en gran medida a los criterios que sus padres tienen sobre un estado socioeconómico superior al promedio.

\section{Colegios estatales}

Los colegios estatales en Alemania pueden seguir filosofías pedagógicas y métodos de enseñanza diferentes. Aunque parece que estos colegios son más o menos exclusivos, es un hecho de que se están convirtiendo cada vez más multiculturales, y así, en contraste a los colegios cristianos y Waldorf, estos no caracterizan un enfoque filosófico o religioso.

\section{Características del estudio para identificar el grado de egocentrismo}

Los cuestionarios estandarizados fueron distribuidos a 521 estudiantes de ocho colegios ubicados en la parte occidental de Alemania (Dortmund, Hagen, Haltern, Olfen, Essen y Schwerte) y dos en Suiza (Basel y Bern). La edad promedio de los participantes fue de 16.6. Del grupo, 53\% eran del género femenino. La mayoría de los participantes se identificó en el grupo de denominación cristiana (53\% católicos, $29 \%$ protestantes), $6 \%$ otras denominaciones, y $12 \%$ ninguna. La mayoría vivía con ambos padres (64\%), 20\% con la madre, $3 \%$ con el padre, $2 \%$ con otra persona, y $11 \%$ no especificó.

Tres colegios públicos $(\mathrm{n}=139)$, dos colegios cristianos $(\mathrm{n}=196)$ y cinco colegios Waldorf $(\mathrm{n}=186)$ participaron en este estudio. La denominación religiosa difiere significativamente en los tres contextos escolares. En los colegios Waldorf, hubo mayor número de protestantes $(38 \%)$ y estudiantes sin denominación religiosa (29\%), mientras que en los colegios cristianos, el grupo mayor fue 
de católicos $(72 \%)$ y de protestantes $(26 \%)$. En los colegios públicos también hubo mayor número en el grupo de católicos $(64 \%)$ y de protestantes $(24 \%)$.

La identificación del grado de egocentrismo de los adolescentes fue basada en el dominio "La consideración hacia los demás está muy bien. Sin embargo, lo que importa en última instancia es que salga adelante en la escuela y en mi carrera", el cual fue previamente evaluado en un estudio piloto. (BÜSSING, 2010a). Teniendo en cuenta que la mutua consideración es socialmente apreciada y deseada, cuando se preguntó directamente si ellos responderían (cognitivamente) de manera positiva, el dominio fue parafraseado indirectamente y evaluado con una escala de tres puntos ( 0 : en desacuerdo, 1: neutral, 2: de acuerdo).

Se usaron dominios individuales para analizar el imperativo moral cognitivo de trabajo para el desarrollo de la sociedad (M1 "Me siento responsable por el desarrollo moral de la sociedad") y la actitud altruista (T4 "Aún si a otros no les parece, me aferro a la idea de ayudar cuanto sea posible"), y en específico al compromiso por ayudar (S25 "Me ofrezco a ayudar a otros"). Estos dominios fueron evaluados en una escala de 5 ( 0 : totalmente en desacuerdo, 1: en desacuerdo, 2: neutral, 3: de acuerdo, 4: completamente de acuerdo).

El dominio de eudemonismo fue explorado en las frases U3 "Todo en la vida se reduce al bienestar de sí mismo" y los dominios de lástima/compasión fueron explorados con $\mathrm{T} 1$ "El ideal de lástima/compasión no encaja en la sociedad moderna" y T2 "Lástima/compasión previenen a otros de cuidar activamente de sus propias necesidades", ambos relacionados con la filosofía de Nietzsche.

Para medir la amplia variedad de los aspectos importantes de la espiritualidad más allá de las fronteras conceptuales convencionales, se desarrolló el cuestionario ASP (Aspectos de espiritualidad, por BÜSSING y colegas, 2007). Para el análisis de este estudio, se emplearon 25 dominios del cuestionario ASP 2.1 , evaluados en un estudio de estudiantes de admisión a la educación religiosa en colegios de bachillerato. (BÜSSING, 2010b). El cuestionario diferencia la orientación religiosa: Oración/confianza en Dios (enfoque religioso; Cronbach's alpha $=.93$ ), la búsqueda interior/sabiduría (percepciones filosóficas/existenciales; alpha $=.88$ ), y las interacciones conscientes (percepciones humanistas; alpha $=.83$ ) y la convicción transcendental (percepciones esotéricas; alpha $=85$ ).

El término Dios fue empleado sólo una vez. El marcador S32 "Me identifico con las normas éticas" y S25 "Ofrezco mi tiempo para trabajar por los demás" fueron tomados desde la versión primaria del cuestionario. Todos los dominios fueron evaluados en una escala de 5 ( 0 : totalmente en desacuerdo; 1 : en desacuerdo; 2: neutral; 3 : de acuerdo; 4: completamente de acuerdo). 


\section{Resultados sobre el posicionamiento egocentrismo}

El egocentrismo fue expresado significativamente menor en el grupo femenino (49\%) que en el masculino. No hubo diferencias significativas que se suscribieran a los tipos de contextos escolares, pero se evidenció una tendencia al nivel de familia (resultados más bajos en los estudiantes que viven con ambos padres) o con denominación religiosa (resultados más altos en estudiantes pertenecientes a la denominación cristiana).

La frase relacionada con la idea de ayudar a los demás tanto como sea posible fue expresada en un $51 \%$ por los estudiantes y rechazada por el $17 \%$, con un $32 \%$ que se mantuvo indeciso. El grupo femenino tuvo un resultado significativamente más alto en este punto, mientras que no hubo diferencias significativas entre los diferentes tipos de colegio, condición familiar o denominación religiosa. Con respecto al compromiso voluntario hacia los demás, $43 \%$ manifestó estar comprometido, $42 \%$ no, y 15\% permaneció indeciso. En esta línea de tendencia, los resultados más bajos fueron hallados en el grupo masculino y en los estudiantes sin denominación religiosa.

La frase relacionada con el sentimiento de responsabilidad por el desarrollo moral de la sociedad fue manifestada por el $29 \%$ de los estudiantes, rechazada por el 33\% y el $38 \%$ permaneció indeciso. No hubo diferencias significativas entre los dos géneros, situación familiar o denominación religiosa. Sin embargo, los estudiantes de los colegios Waldorf presentaron los resultados más altos al respecto.

\section{Análisis de correlación entre egocentrismo, ideales éticos, aspectos de espiritualidad y satisfacción de vida}

El egocentrismo se correlacionó moderadamente $(r<0.4)$ con el eudemonismo, ligeramente positivo $(\mathrm{r}<0.3)$ con las frases negativas en relación con el tema de compasión (T1 y T2), y ligeramente negativo en relación con el ideal de ayudar a otros (T4) y el trabajo voluntario (S25). El posicionamiento egocéntrico se correlacionó ligeramente de manera negativa con aspectos de espiritualidad, particularmente con Compasión/Generosidad y Orientación, pero no con la satisfacción de vida.

El ideal de ayudar a otros (S4) fue correlacionado moderadamente con Compasión/Generosidad, y correlacionado ligeramente con otros aspectos de 
espiritualidad, interés en ayudar a otros (S25) y eudemonismo (U3), pero no correlacionado con compasión o satisfacción de vida.

En una investigación previa, la actitud de posicionamiento fue rechazada por la mayoría del grupo femenino. (BÜSSING, 2010b). La intención de este trabajo exploratorio fue más allá de analizar las actitudes que pueden estar asociadas con el posicionamiento egocéntrico en el contexto de compasión y eudemonismo, las asociaciones con aspectos de espiritualidad y la influencia del contexto escolar.

Los hallazgos de este estudio confirman que tanto el posicionamiento de egocentrismo como la idea de ayudar a los demás fueron de relevancia superior en el grupo femenino que en el masculino. Sin embargo, el tipo de contexto escolar no presentó impacto significativo sobre estas actitudes. Análisis de regresión de variables indicaron que la actitud de egocentrismo puede ser identificada mejor a través de dos indicadores: el eudemonismo y la convicción de que la compasión por los demás previene tomar iniciativas propias.

Con respecto a las ideas positivas como ayudar a otros, ofrecerse como voluntario, y la convicción de ser responsable por la sociedad, fue evidente que la Compasión/Generosidad fueron los mejores predictores, modulados por el grupo de variables ${ }^{4}$. A este respecto, el tipo de colegio no tuvo (entre las variables) una influencia significativa. Sin embargo, esto no va en contra del hecho de que el sistema educativo cultiva actitudes importantes durante el desarrollo de la adolescencia. De hecho, las actitudes subyacentes de los adolescentes son importantes, no por los tipos de escuela por sí mismos, aunque estas pueden motivar posiciones éticas bien definidas.

Los estudiantes de colegios Waldorf parecen estar más decididos en sus respuestas porque todas las correlaciones fueron significativamente mayores que en los otros grupos. Estos efectos no pueden ser explicados por diferencias significativas con respecto al posicionamiento egocéntrico, la satisfacción de vida, las interacciones conscientes o de Compasión/Generosidad, porque estas no difieren. Se puede afirmar que la orientación y la convicción transcendente fueron expresadas significativamente mayores en los estudiantes de los colegios Waldorf, aunque ellos no estuvieron entre los predictores significativos.

Como conclusión preliminar, se puede argumentar que los adolescentes valoran sus ideales positivos. El tipo de colegio, las características familiares y la afiliación religiosa son variables importantes que moldean en particular la Compasión y Generosidad. Schopenhauer (2011) argumenta que la compasión (o lástima) es la virtud más grande y la base única de la moralidad genuina,

4 El posicionamiento altruista y espiritual de los adolescentes fue investigado en el área de la educación alta en colegios cristianos en Alemania. (BÜSSING et al., 2012). 
porque esta debería ser capaz de sobrepasar las inclinaciones egoístas y motivar la actuación del bienestar de los demás. Si esta actitud es activamente promovida por los colegios participantes o por el ambiente familiar de los adolescentes es un tema debatible a investigar.

Ciertos aspectos de espiritualidad fueron asociados con ideales positivos, mientras que el posicionamiento egocéntrico fue sólo ligeramente influenciado por actitudes espirituales, en este aspecto, la satisfacción con la situación escolar fue de gran relevancia. Esto significa que los estudiantes se comportan diferente de acuerdo con la satisfacción escolar, la cual puede implicar notas, docentes, compañeros, perspectivas futuras, etc.

De particular intereses es el hecho de que la religiosidad convencional es de importancia solamente para los estudiantes que consideran la opción de ofrecerse a colaborar, mientras que el humanismo secular tuvo una influencia más general. De hecho, la religiosidad convencional fue un factor de relevancia inferior para todos los estudiantes. Esto es enfatizado por Anton Bucher (2009a), citando los hallazgos de Ziebertz (2008):

\begin{abstract}
'Una idea generalizada sobre los jóvenes y adultos jóvenes persiste afirmando que, en esta fase de su vida, su apego a las comunidades religiosas se vuelve más flexible, su acuerdo con las creencias religiosas más tentativas, y la religiosidad en general menos relevante para sus vidas.' 'Casi cada era se ha unido en una elegía lamentando el hecho de que la nueva generación no ha interiorizado lo suficiente el corpus de las convicciones religiosas heredadas, lo que resulta en el abandono de las tradiciones religiosas y la propagación de la secularización [...]. (BUCHER, 2009a, p. 607).
\end{abstract}

Esto coincide con los resultados del Estudio de la Juventud Shell (número 15), que afirma que "la religiosidad de afiliación a la iglesia tiende a exagerar el perfil del valor normal de la juventud de hoy con respecto a un subgrupo especial de adolescentes en virtud de su mayor orientación hacia la familia, las normas sociales, la salud y el compromiso social." (GENSICKE, 2006). Sin embargo, el autor subraya que esto se explica fácilmente por los contenidos religiosos o sus consecuencias. Esto apunta al impacto específico del contexto cultural y sus contenidos inherentes a la selección de valores.

Estos hallazgos confirman que los aspectos eclesiásticos de religiosidad son menos importantes, mientras que los aspectos conectivos de espiritualidad (interacciones conscientes, Compasión/Generosidad) fueron de alta relevan- 
cia. Bucher (2009a) ofrece una interpretación similar. Habiendo examinado los estudios cualitativos relevantes sobre tradiciones religioso-espirituales, él ve un motivo básico recurrente, por ejemplo, la afinidad. (BUCHER, 2009a). Este valor parece ser un dato empírico de la vida espiritual y la religiosidad intrínseca y su aplicación es independiente de los antecedentes culturales o la situación geográfica del espiritualmente activo. En una visión diferenciada de este concepto, dos posibles dimensiones de afiliación aparecen: "Uno se extiende verticalmente hacia Dios, la trascendencia, un ser superior; el otro horizontal, hacia el prójimo, la naturaleza, e incluso todo el cosmos." (BUCHER, 2009a). Estas orientaciones de afinidad no tienen que ser imaginadas mutuamente excluyentes, sino más bien como un complemento entre sí que forma de esta manera un todo unificado. El 'centro de acción' de afinidad espiritual es la conciencia humana, con "autotrascendencia" que representa una característica clave. Dejar ir la propia subjetividad es una necesidad interior si la experiencia espiritual de afinidad toma lugar en los diferentes niveles del ser.

FIGURA 1 - Modelo de espiritualidad

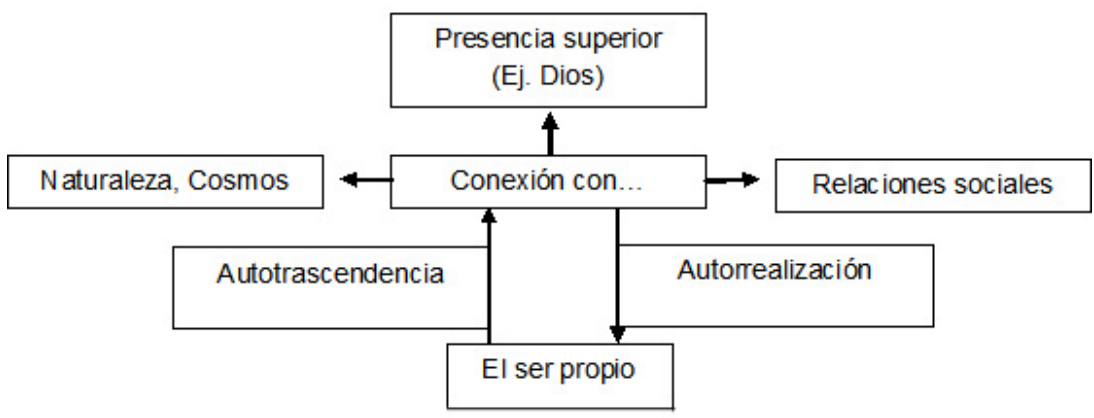

FUENTE: Modificado de acuerdo con BUCHER (2009a, p. 16).

Sin duda, es posible integrar la religiosidad o una vida religiosa activa en este modelo. En la literatura, sin embargo, la espiritualidad tiende a conceder un mayor grado de independencia con respecto a las formas institucionalizadas de actividad espiritual, haciendo hincapié en la "primacía de la propia experiencia de la trascendencia de uno y la meditación”. (ZWINGMANN, 2009, p. 186).

En conclusión, este estudio analizó más a fondo las actitudes subyacentes de los adolescentes asociadas con posiciones egocéntricas y tendencias altruistas, en relación a factores como la escolaridad y la espiritualidad. Los resultados son representativos, puesto que no hay indicios fuertes de que las formas escolares investigadas tengan un impacto por sí mismos. 
Se encontró que los individuos (o sus padres, respectivamente) con actitudes o convicciones específicas eligen el tipo de escuela que esté en comunión con su ideología (selección ideológica, social y económica). Sin embargo, se identificaron distintos aspectos de la espiritualidad como predictores significativos de tendencias altruistas, mientras que las posiciones egocéntricas pueden predecirse a través de una actitud de eudemonismo, que es modulada negativamente por la satisfacción con la situación de la escuela.

Esto indica que la escuela misma puede tener un impacto significativo en el compromiso ético de los estudiantes y puede contribuir en el proceso de desarrollo de los adolescentes a adultos como seres sociales y morales, con una responsabilidad hacia el medio ambiente, la economía y la sociedad. La pregunta sobre cómo este proceso de "llegar a ser" se podría estimular y fomentar requiere mayor investigación.

Una limitación de este enfoque es que los estados cognitivos de los estudiantes podrían no coincidir con su comportamiento real. Por otra parte, este estudio no pretendió dar una imagen representativa del sistema escolar de Alemania. La intención no era argumentar a favor o en contra de un contexto escolar específico, sino referirse al trasfondo (religioso o filosófico) de diferentes formas escolares que pueden tener un impacto diferente en las actitudes y valores de los adolescentes. De hecho, otros estudios que incluyen más contextos escolares convencionales están actualmente en marcha.

\section{REFERENCIAS}

BAIER, D. K. F. N. Forschungsbericht Nr. 104. Entwicklung der Jugenddelinquenz und ausgewählter Bedingungsfaktoren seit 1998 in den Städten Hannover, München, Stuttgart und Schwäbisch Gmünd. Hannover: Kriminologisches Institut Niedersachsen, 2008.

BARZ, H.; RANDOLL, D. (Eds.). Absolventen von Waldorfschulen. Eine empirische Studie zu Bildung und Lebensgestaltung. Wiesbaden: VS, 2007.

BAUMERT, J.; KOELLER, O. Nationale und internationale Schulleistungsstudien: Was können sie leisten, wo sind ihre Grenzen? Pädagogik, v. 50, p. 12-18, 1998.

BAUMERT, J.; KOELLER, O.; SCHNABEL, K. Schulformen als differentielle Entwicklungsmilieus - eine ungehörige Fragestellung? Erwiderung auf die Expertise "Zur Messung sozialer Motivation in der BIJU-Studie" von Georg Lind. Disponible en: $<$ http:// www.mpib-berlin.mpg.de/en/institut/dok/full/Baumert/schulfor/lind.pdf $>$. Acceso en: 3 abr. 2015. 
BERGER, P. L.; BERGER, B.; KELLNER, H. Das Unbehagen in der Modernität. Frankfurt a.M.: New York: Campus, 1975.

BEYER, H. F. Religion unterrichten in NRW. Theo-Web. Zeitschrift für Religionspädagogik, v. 5, n. 2, p. 179-187, 2006.

BOBERT, S. Autonom spirituell sein - Protestantische Spiritualität zwischen Atomisierung und Stellvertretung. Vortrag zu: Spiritualität - Baustein oder Stolperstein für die Kirche? Eine Tagung zum Spannungsfeld von Aszetik und Kybernetik im interkonfessionellen Gespräch, Zürich, 9/10 Jul. 2007. Disponible en: <http://www.theologie.uzh.ch/ faecher/praktisch/ralph-kunz/Bobert_Autonom_spirituell.pdf $>$. Acceso en: 29 mar. 2015.

BONHOEFFER, A.; BRATER, M.; HEMMER-SCHANZE, C. Berufliche Entwicklung ehemaliger Waldorfschüler. In: BARZ, H.; RANDOLL, D. Absolventen von Waldorfschulen. Eine empirische Studie zu Bildung und Lebensgestaltung. Wiesbaden: VS, 1997. p. 45-99.

BOURDIEU, P. Ökonomisches Kapital, kulturelles Kapital, soziales Kapital. In: KRECKEL, L. (Ed.). Soziale Ungleichheiten. Göttingen: Schwartz (Reihe Soziale Welt Sonderband II), 1983. p. 183-198.

BRINKHOFF, K.-P.; FERCHOFF, W. Jugend und Sport. Zur Karriere einer offenen Zweierbeziehung. In: HEITMEYER, W.; OLK, T. (Eds.). Individualisierung von Jugend: Gesellschaftliche Prozesse, subjektive Verarbeitungsformen, jugendpolitische Konsequenzen. Weinheim und München: Juventa Verlag, 1990. p. 99-129.

BUCHER, A. Religiosity and Spirituality among Young Adults. In: BERTELSMANN STIFTUNG (Ed.). What the World Believes? Analyses and Commentary on the Religion Monitor, Gütersloh: Verlag Bertelsmann Stiftung. 2009a. p. 607-627.

BUCHER, A. Empirische Psychologie der Spiritualität: Möglichkeiten und Grenzen - ein Forschungsüberblick. In: REITER, A.; BUCHER, A. (Eds.). Psychologie - Spiritualität - interdisziplinär. Eschborn: Dietmar Klotz, 2009b. p. 8-32.

BUND DER FREIEN WALDORFSCHULEN. Waldorf and Rudolf Steiner Schools and Teacher Training Centers worldwide. 2014. Disponible en: $<$ http://www.freunde-waldorf. de/fileadmin/user_upload/images/Waldorf_World_List/Waldorf_World_List.pdf, 2014>. Acceso en: 3 abr. 2015.

BÜSSING, A. Aspects of spirituality, God images, and the 'self-centredness' in 17 year old adolescents attending religious education at high-school. RoSE-Research on Steiner Education, v. 1, p. 75-84, 2010a.

BÜSSING, A. Die Bedeutung von Religiosität und Spiritualität für chronisch Kranke. In: BALCK, F.; BERTH, H.; KLEIN, C. Die Bedeutung von Religion für die Gesundheit. Konzepte - Befunde - Erklärungsansätze. Weinheim: Juventa Verlag, $2010 \mathrm{~b}$.

BÜSSING, A. "Spiritualität" - Worüber reden wir? In: BÜSSING, A.; OSTERMANN, T.; GLÖCKLER, M.; MATTHIESSEN, P. Spiritualität, Krankheit und Heilung - Be- 
deutung und Ausdrucksformen der Spiritualität in der Medizin. Frankfurt: VAS - Verlag für Akademische Schriften, 2006. p. 11-24.

BÜSSING, A.; FISCHER, J.; HALLER, A.; OSTERMANN, T.; MATTHIESSEN, P. F. Validation of the Brief Multidimensional Life Satisfaction Scale in patients with chronic diseases. European Journal of Medical Research, v. 14, p. 171-177, 2009.

BÜSSING, A.; KERKSIECK, P.; FÖLLER-MANCINI, A.; BAUMANN, K. Aspects of Spirituality and Ideals of Help in Adolescents from Christian Academic High Schools. International Journal of Children's Spirituality. DOI:10.1080/1364436X.2012.680882. 2012.

BÜSSING, A.; OSTERMANN, T.; MATTHIESSEN, P. F. Distinct expressions of vital spirituality. The ASP questionnaire as an explorative research tool. Journal of Religion and Health, v. 46, p. 267-286, 2007.

DIE DEUTSCHEN BISCHÖFE. (Eds.). Qualitätskriterien für Katholische Schulen. Ein Orientierungsrahmen. Bonn: Sekretariat der Deutschen Bischofskonferenz. 2009. n. 90.

ERIKSON, E. Identität und Lebenszyklus. Frankfurt: Suhrkamp, 1973.

FEND, H. Sozialisationseffekte der Schule. Soziologie der Schule, v. II. Weinheim und Basel: Beltz, 1976.

GENSICKE, T. Jugend und Religiosität. In: HURRELMANN, K.; ALBERT, M. (Eds.). 15. Shell-Jugendstudie. Frankfurt/M.: Fischer Verlag, 2006. p. 203-239.

GENSICKE, T. Lebensorientierungen. In: BARZ, R.; RANDOLL, D. (Eds.). Absolventen von Waldorfschulen. Eine empirische Studie zu Bildung und Lebensgestaltung. Wiesbaden: VS, 2007. p. 101-131.

HEIMBACH-STEINS, M. Das Menschenrecht auf Bildung: Ein Maßstab für den sozialen Bildungsauftrag katholischer Schulen. Vortrag, veröffentlicht im Rahmen einer Pressemitteilung der Deutschen Bischofskonferenz am 28.11.2008.

HOOCK, S. Der Trend geht zum Privat-Gymnasium. Der Westen, p. 1, 2010, 2. Januar. Disponible en: <http://www.derwesten.de/waz-info/der-trend-geht-zum-privat-gymnasium-id2333260.html>. Acceso en: 30 mar. 2015.

HURRELMANN, K. Lebensphase Jugend. Eine Einführung in die sozialwissenschaftliche Jugendforschung. Weinheim, München: Juventa, 2010.

INGLEHART, R. Kultureller Umbruch. Wertwandel in der westlichen Welt. Frankfurt/M.: New York: Campus, 1998a.

INGLEHART, R. Modernisierung und Postmodernisierung. Kultureller, wirtschaftlicher und politischer Wandel in 43 Gesellschaften. Frankfurt/M.: New York: Campus, 1998b.

MANSELL, J. Sozialisation in der Risikogesellschaft. Eine Untersuchung zu psychosozialen Belastungen Jugendlicher als Folge ihrer Bewertung gesellschaftlicher Bedrohungspotentiale. Neuwied: Luchterhand, 1995. 
OSTERMANN, T.; BÜSSING, A. Spiritualität und Gesundheit: Konzepte, Operationalisierung, Studienergebnisse. Musiktherapeutische Umschau, v. 28, n. 3, p. 217-230, 2007.

RANDOLL, D. Empirische Forschung und Waldorfpädagogik. In: PASCHEN, H. (Ed.). Erziehungswissenschaftliche Zugänge zur Waldorfpädagogik. Wiesbaden: Verlag für Sozialwissenschaften, 2010.

RANDOLL, D. "Schulwirklichkeiten". Vergleichende Betrachtung der Wahrnehmung von Schule durch Abiturienten und ihren Lehrern. Baden: Nomos, 1997.

ROLFF, H. Sozialisation und Auslese durch die Schule. Weinheim und München: Juventa, 1997.

SCHIEREN, J. Die Veranlagung von intuitiven Fähigkeiten in der Pädagogik. RoSE Research on Steiner Education, v. 1, p. 7-18, 2010.

SCHOPENHAUER, A. Über das Mitleid. München: dtv, 2011.

SEYRINGER, M.-E.; FRIEDRICH, F.; STOMPE, T.; FROTTIER, P.; SCHRANK, B.; FRÜHWALD, S. Die "Gretchenfrage" für die Psychiatrie. Der Stellenwert von Religion und Spiritualität in der Behandlung psychisch Kranker. Neuropsychiatrie, v. 21, n. 4, p. 239-247, 2007.

STANDFEST, C.; KÖLLER, O.; SCHEUNPFLUG, A.; WEISS, M. Profil und Erträge von evangelischen und katholischen Schulen. Befunde aus Sekundäranalysen der PISADaten. Zeitschrift für Erziehungswissenschaft, v. 3, p. 359-379, 2004.

ZIEBERTZ, H.-G. Gibt es einen Tradierungsbruch? Befunde zur Religiosität der jungen Generation. In: BERTELSMANN STIFTUNG (Ed.). Religionsmonitor 2008. Gütersloh: Bertelsmann Verlag, 2007. p. 44-53.

ZINNECKER, J. Selbstsozialisation - Essay über ein aktuelles Konzept. Zeitschrift für Sozialisationsforschung und Erziehungssoziologie, v. 20, p. 272-290, 2000.

ZWINGMANN, Ch. Spritualität und Religiosität als Ressource zur Bewältigung einer chronischen Erkrankung? Perspektiven für Rehabilitation und Psychotherapie, 2009. Disponible en: <http://www.akademieps.de>. Acceso en: 12 jul. 2010.

Texto recebido em 22 de maio de 2015. Texto aprovado em 10 de junho de 2015. 
